\title{
An experimental millimeter wave radio over fiber link with double polarization multiplexing
}

\author{
Nada Badraoui ${ }^{1} \cdot$ Tibor Berceli $^{1}$ (D) \\ Received: 31 January 2021 / Accepted: 3 August 2021 / Published online: 18 August 2021 \\ (c) The Author(s) 2021
}

\begin{abstract}
A novel approach, an optical radio over fiber link with double polarization multiplexing is presented. In the two orthogonal polarizations of the optical beam the contents of information to be transmitted are different doubling the link capacity this way. For experimental verification a millimeter wave radio over fiber link with double polarization multiplexing has been developed. To ensure low cross polarization an incoherent approach is used. A high quality signal transmission is achieved by a proper procedure providing high polarization extinction ratio. In the experimental investigations different bit rates and fiber lengths are used. A signal with $12 \mathrm{Gbit} / \mathrm{s}$ bit rate is transmitted over a $25 \mathrm{~km}$ long link with about $1.10^{-8}$ bit error rate. That result is much better than the already published data measured on experimental links.
\end{abstract}

Keywords Double orthogonal polarization - Cross polarization · Polarization extinction ratio $\cdot$ Polarization multiplexin

\section{Introduction}

The increasing demand for improving the service of present $5 \mathrm{G}$ and future $6 \mathrm{G}$ mobile networks requires signal transmission with higher capacity. The radio over fiber (RoF) link provides proper connections between a center station and its radio base stations in mobile networks.

Increasing the capacity of existing optical links is an important issue (Sekine et al. 1995). Presently for that, the polarization division multiplex (PDM) method is applied together with wavelength division multiplex (WDM) (Hayee et al. 2001; Perez et al. 2009). In that approach the optical beams at each wavelength have only one polarization, while the polarization of the adjacent channels is orthogonal. This way the wavelength difference between the adjacent channels can be reduced resulting in modest capacity enhancement.

Nada Badraoui

nada@hvt.bme.hu

Tibor Berceli

berceli.tibor@vik.bme.hu

1 Budapest University of Technology and Economics, Budapest, Hungary 
The polarization division multiplexing technique has already been investigated in some publications (Perez et al. 2009; Morant et al. 2014; Yao et al. 2007). Most of them were theoretical studies or simulations (Core 2006). However, the combination of polarization division multiplex and wavelength division multiplex is a complex procedure, therefore it needs a relatively sophisticated system architecture.

Most of the papers considered applications for short distances at low radio frequency (Johny et al. 2012; Yoshida et al. 2016), typically in the $2 \mathrm{GHz}$ band with relatively low bit rates, usually $2.5 \mathrm{Gbit} / \mathrm{s}$. However, in advanced systems higher bit rates are required. To achieve that goal the used radio frequency should be pushed to higher frequencies, mainly into the millimeter wave band.

In the present paper a novel approach, the double polarization multiplex (DPM) method is presented and investigated. In that case the optical beam has simultaneously two orthogonal polarizations or by other words it has double polarization. In the orthogonal polarizations of the beam the contents of information to be transmitted are different. The simulation results of the double polarization multiplexing approach in radio over fiber links are promising (Badraoui and Berceli 2018, 2019, 2020). However, experimental investigations on these links have not been done in details yet.

An experimental link is presented in this paper to validate the usefulness and advantage of the double polarization multiplex approach applied in radio over fiber links. The polarization multiplexing method having two orthogonal polarizations at the same optical wavelength is introduced to double the capacity by transmitting two channels with different information (Badraoui and Berceli 2018, 2019, 2020). In this application the cross polarization between the two polarizations has to be kept at a specified low level to avoid cross modulation between the two channels. The aim of the present experiment is to validate the applicability of the new method in advanced mobile networks providing increased capacity for the links connecting the center station and the radio base stations.

There are two procedures to generate the optical wave with two orthogonal polarizations. In one approach a common optical source is applied for creating two optical information transmitting channels such a way that the optical beam is generated by a common laser source and it is divided in two equal parts. That means the two optical beams are coherent. In the other approach two independent optical sources are applied with the same wavelength. That means the two optical beams are incoherent. The incoherency helps to reduce the effect of cross modulation between the two channels having orthogonal polarization. In the following the incoherent approach is applied and investigated. In both cases the independence of the channels is only based on the polarization difference, i.e. on the polarization orthogonality. That means there is no need for optical filtering to combine and separate the two channels. That is a big advantage of the double polarization multiplexing method.

\section{Experimental millimeter wave radio over fiber link with double polarization multiplexing}

An experimental radio over fiber link using double polarization multiplexing has been developed and tested in the laboratory. Two independent channels were created by applying double polarization multiplexing. For this an optical beam was generated with very high polarization extinction ratio (PER). Based on our previous simulation results (Badraoui and Berceli 2019) we need at least $22 \mathrm{~dB}$ polarization extinction ratio (PER) in the 
transmitter. That means the intensity of the used polarization has to be $22 \mathrm{~dB}$ higher than that of the orthogonal polarization of the optical beam. That goal can be obtained by a suitable procedure.

The emitted laser beams usually have elliptical polarization. Therefore polarization controller is applied to get pure linear polarization by eliminating the smaller perpendicular polarization. The laser beam is passed through a linear polarizer plate in the polarization controller type PC-HP 8169A to create linear polarization. Then by the quarter wave $(\lambda / 4)$ and half wave $(\lambda / 2)$ plates the state of polarization $(\mathrm{SoP})$ is adjusted. This way two independent orthogonal polarization states can be generated from the beam having pure linear polarization.

\subsection{Adjustment of polarization states}

The first step is the adjustment of polarization states. That is shown in Fig. 1. The outputs of laser 1 and laser 2 are connected to a $3 \mathrm{~dB}$ directional coupler. This way we get their beams at the same output of the $3 \mathrm{~dB}$ directional coupler simultaneously. When the beam of laser 1 is adjusted, laser 2 is switched off. Similarly when the beam of laser 2 is adjusted, then laser 1 is switched off.

In the next step the beam of laser 1 with linear polarization is rotated by the polarization controller 1 (PC 1) to get maximum intensity at the $\mathrm{X}$ output of polarization beam splitter (PBS) and minimum at its $\mathrm{Y}$ output. There is $90^{\circ}$ polarization angle difference between the two outputs. The same adjustment is done with the beam of laser 2. However, in that case by using polarization controller 2 (PC 2), minimum intensity has to be adjusted at the $\mathrm{X}$ output of polarization beam splitter and maximum of its $\mathrm{Y}$ output. With this procedure we get two optical beams with orthogonal polarization. Using this method we achieved $25 \mathrm{~dB}$ polarization extinction ratios in both cases. The polarization of the optical beams is adjusted with the same procedure also in the other parts of the transmitter. This way we kept $25 \mathrm{~dB}$ polarization extinction ratio at the transmitter output as well.

\subsection{Independence of channels with double polarization}

The experimental investigations intended to validate the expected behavior of the double polarization multiplex method. In the first step the independence of the two orthogonal polarization states was investigated by suitable experiments. The measurements on an

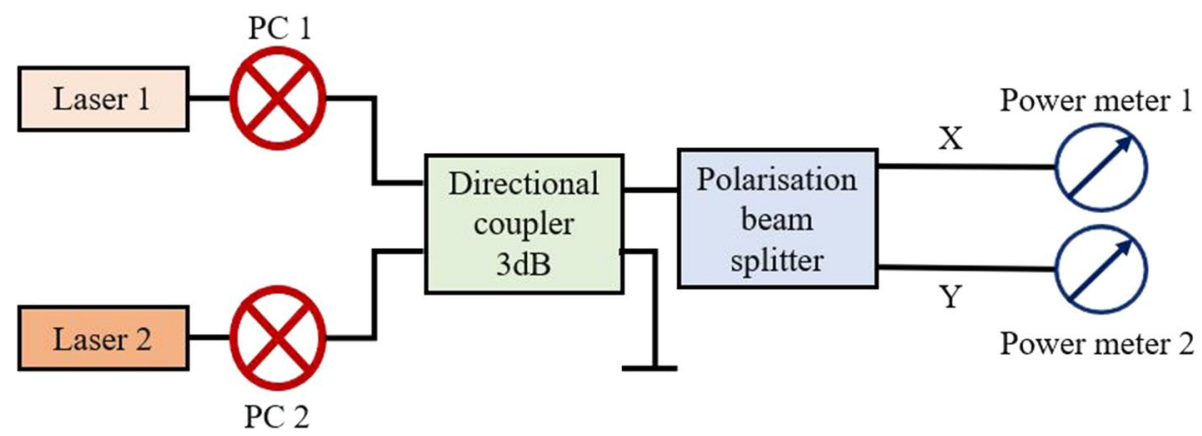

Fig. 1 Polarization adjustment procedure 
experimental set-up were performed with the aim to prove that the two orthogonal polarization states are independent. That means there is no coupling, therefore no cross modulation between them.

The block diagram of Fig. 2 shows the experimental set-up. In that there are two optical sources with the same frequency: $193.5 \mathrm{THz}$. The laser frequencies were stabilized. The two optical beams are modulated by two random data sequences in Mach-Zehnder optical modulators. This way the optical links have modulation in the baseband. The two modulated beams are adjusted by two polarization controllers to orthogonal polarization states as that is described in chapter 2.1. Then a polarization beam combiner (PBC) produces the combined beam with two orthogonal polarizations. That optical beam is transmitted over a single mode fiber (SMF) to the reception side. In the receiver a polarization beam splitter (PBS) separates the two polarizations.

In the experiments the Corning SMF-28e type fiber has been used which has a relatively low chromatic dispersion coefficient at $1.55 \mu \mathrm{m}$ wavelength: $\mathrm{D}=12.5 \mathrm{ps} /(\mathrm{nm} . \mathrm{km})$, and a small attenuation: $0.2 \mathrm{~dB} / \mathrm{km}$.

These channels have been tested by measuring the bit error rate (BER) and eye diagram (EYE). In the experiment a common clock was used for the data sequences. This way the channels are synchronous which means a worst case test.

The notations are the following: $\mathrm{PC}=$ polarization controller, $\mathrm{MZM}=$ Mach Zehnder modulator, $\mathrm{PBC}=$ polarization beam combiner, $\mathrm{PBS}=$ polarization beam splitter, $\mathrm{SMF}$ $=$ single mode fiber, $\mathrm{PhD}=$ photo diode, $\mathrm{Ch}=$ channel, $\mathrm{BER}=$ bit error rate, $\mathrm{EYE}=$ eye diagram.

According to the block diagram we tested both channels separately. That means in the first step channel 1 had no modulation on the beam of laser 1 which is called OFF state. At the same time channel 2 had modulation on the beam of laser 2 which is called ON state. In this case at the reception side of channel 1 we had only noise as can be seen in Table 1 .

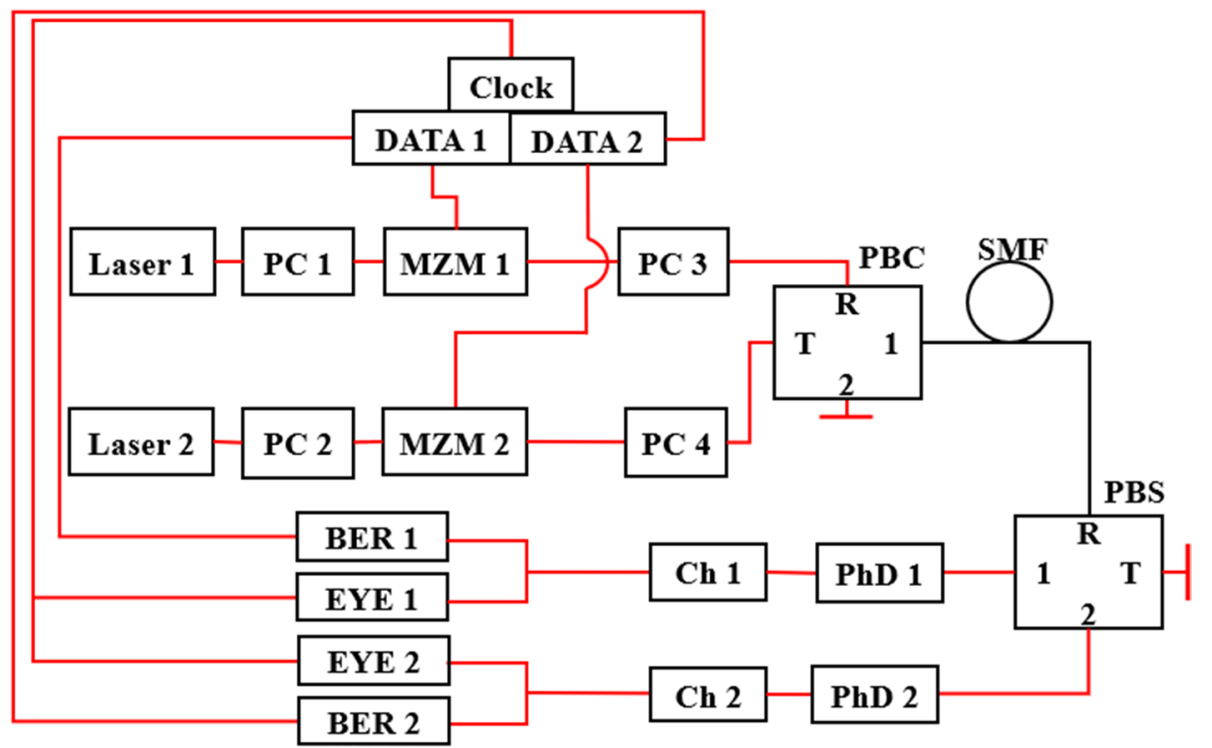

Fig. 2 Block diagram of the experiment for checking the independency of channels with orthogonal polarization 
Table 1 Measurement results of the base band optical link with double polarization multiplexing

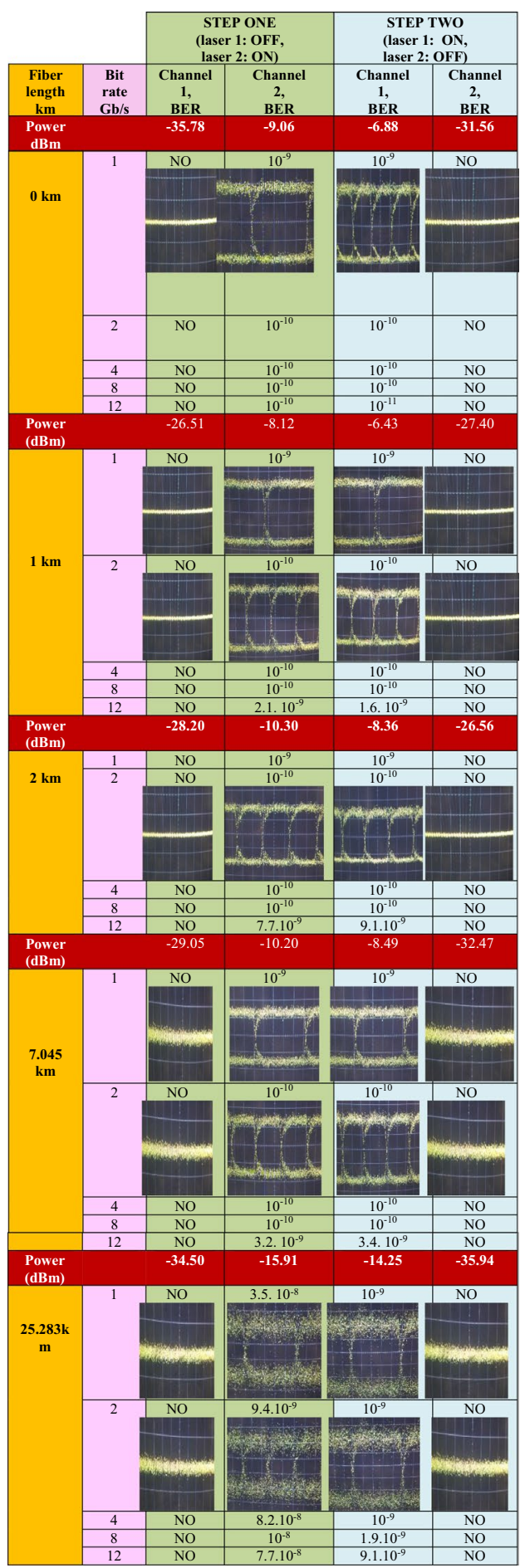


The receiver of channel 1 was in operation, however, not obtaining any modulation, the bit error rate measurement was not possible. That proves that no cross modulation was obtained from channel 2 . Then we investigated the opposite version when channel 1 was in ON state, i.e. it had a modulation signal and channel 2 had no modulation signal, i.e. it was on OFF state.

We obtained now only noise in channel 2 at the reception side and no detected signal from channel 1. The receiver of channel 2 was in operation, however, not having any modulation, the bit error rate measurement was not possible. That proves that no cross modulation was obtained from channel 1 .

The above cases were checked first without a fiber connection, i.e. in a back-to-back arrangement. As can be seen in Table 1 there was no signal in a channel with state OFF from a channel in state ON. In these cases we observed only noise in the OFF channels. As it is seen in Table 1, the channels in state OFF had no eye diagrams because there were no modulated signals. These measurements were continued using higher bit rates. However, the same results were obtained as in the previous cases. That means there was no cross modulation and consequently no cross polarization between the two channels.

Having done these basic tests with different bit rates the investigation has been extended by applying fiber connections with different lengths between the transmitter and receiver.

We applied different pieces of fibers with lengths of 1,2, 7 and $25 \mathrm{~km}$. In every case the bit rate was varied between 1 and 12 Gbit/s. The eye diagrams were tested along with the measurements of bit error rate. With increasing the fiber length no cross polarization and therefore no cross modulation has been observed between the two orthogonal polarizations. We only observed noise in the received and detected unmodulated channel. According to Table 1 there is no cross polarization effect even when using a $25 \mathrm{~km}$ long fiber with bit rate of $12 \mathrm{Gbit} / \mathrm{s}$.

\subsection{Radio over fiber link with double polarization multiplexing}

The aim of next experiment was to check the transmission properties of the radio over fiber operation with double polarization multiplex. In this experiment both polarizations were used for information transmission. A local oscillator laser beam has also been inserted at the end of the transmitter between the two polarizations with $45^{\circ}$ polarization angle. The local oscillator beam can be decomposed into two parts: one half of its intensity is in the same polarization of channel 1 and the other half of its intensity is in the same polarization of channel 2. The block diagram of that experiment is presented in Fig. 3. As a result two millimeter wave signals are generated at the reception side by mixing the beams of the two laser sources with the beam of the local oscillator laser working at $193.440 \mathrm{THz}$ frequency. The mixing products are then at $60 \mathrm{GHz}$. These millimeter wave signals keeping the original information are amplified and detected for measurements.

The notations are the following: $\mathrm{PC}=$ polarization controller, $\mathrm{MZM}=$ Mach Zehnder modulator, $\mathrm{LO}$ laser $=$ local oscillator laser, $\mathrm{PBC}=$ polarization beam combiner, $\mathrm{PBS}=$ polarization beam splitter, $\mathrm{SMF}=$ single mode fiber, $\mathrm{MMW}=$ millimeter wave, $\mathrm{PhD}=$ photo diode, $\mathrm{Amp}=$ amplifier, Det $=$ detector, BER = bit error rate, EYE = eye diagram.

The measurement results of the millimeter wave radio over fiber link with double polarization multiplexing are presented in Table 2. The millimeter wave radio over fiber link was investigated by measuring the bit error rate and testing the eye diagram. In the experiment different bit rates and fiber lengths were used. Almost the same results are obtained in both channels compared to the previous measurement results presented in Table 1 . The eye 


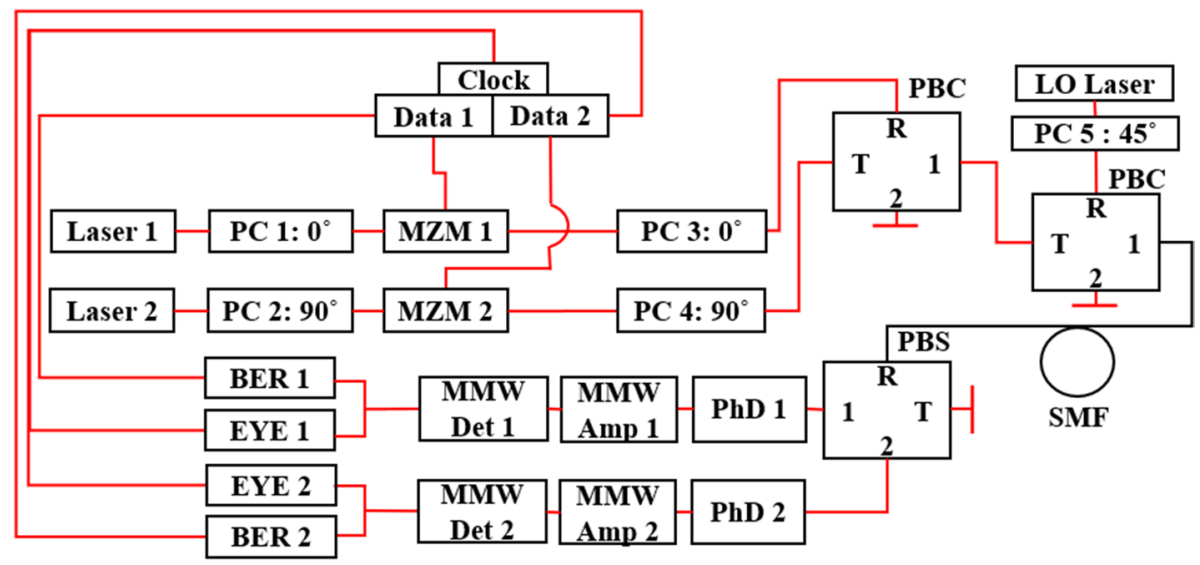

Fig. 3 Block diagram of the radio over fiber link with double polarization multiplexing

diagrams are open also at higher bit rates. They are not presented in every case because no significant change was observed with increasing bit rates. A small deterioration has been obtained both in the bit error rate and eye diagram with increasing bit rate. In spite of that as it can be seen in Table 2, a very good bit error rate of $1.2 .10^{-8}$ was measured even with $25 \mathrm{~km}$ long fiber transmitting a data stream of $12 \mathrm{Gbit} / \mathrm{s}$ bit rate.

It is also worth mentioning that there is no need for optical filtering to combine and separate the two channels. That means the independence of the channels is only based on the polarization difference, i.e. on the polarization orthogonality.

\section{Conclusion}

A novel approach, a radio over fiber link with double polarization multiplexing has been presented. In the two orthogonal polarizations of the optical beam the contents of information to be transmitted are different doubling the link capacity this way. For experimental verification a millimeter wave radio over fiber link with double polarization multiplexing has been developed.

First an optical beam using baseband modulation in its two orthogonal polarizations was investigated experimentally for testing the cross modulation effect between the orthogonal polarizations. Then a millimeter wave radio over fiber link with two orthogonal polarizations was measured for checking its capabilities. It is worth mentioning that the independence of the channels is only based on the polarization difference, i.e. on the polarization orthogonality. That means there is no need for optical filtering to combine and separate the two channels. That function is performed by polarization beam combiners and splitters. In the experimental investigations different bit rates and fiber lengths were used.

A high quality signal transmission has been achieved by a procedure providing $25 \mathrm{~dB}$ polarization extinction ratio. A signal with $12 \mathrm{Gbit} / \mathrm{s}$ bit rate was transmitted over a $25 \mathrm{~km}$ long link with about $1.10^{-8}$ bit error rate in the channels of orthogonal polarizations. 
Table 2 Measurement results of the radio over fiber link with double polarization multiplexing

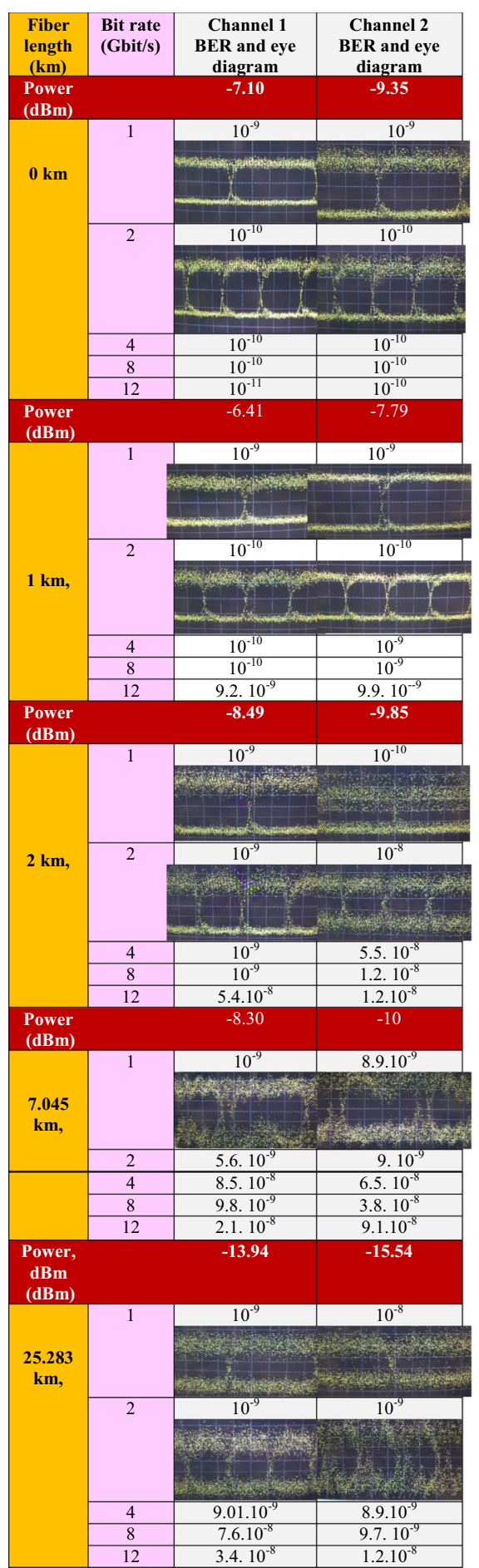


Acknowledgements The authors acknowledge Andreas Stöhr and Matthias Steeg at University of Duisburg, Germany for the excellent consultations. They also acknowledge the COST project CA16220 EUIMWP and K132050 project provided by the National Research, Development and Innovation Office of Hungary for helping their research.

Funding Open access funding provided by Budapest University of Technology and Economics.

Open Access This article is licensed under a Creative Commons Attribution 4.0 International License, which permits use, sharing, adaptation, distribution and reproduction in any medium or format, as long as you give appropriate credit to the original author(s) and the source, provide a link to the Creative Commons licence, and indicate if changes were made. The images or other third party material in this article are included in the article's Creative Commons licence, unless indicated otherwise in a credit line to the material. If material is not included in the article's Creative Commons licence and your intended use is not permitted by statutory regulation or exceeds the permitted use, you will need to obtain permission directly from the copyright holder. To view a copy of this licence, visit http://creativecommons.org/licenses/by/4.0/.

\section{References}

Badraoui, N., Berceli, T.: Behaviour of cross polarization on radio over fiber links. IEEE 11th International Symposium on Communication Systems, Networks \& Digital Signal Processing (CSNDSP), pp. 1-5 (2018)

Badraoui, N., Berceli, T.: Enhancing capacity of optical links using polarization multiplexing. Opt. Quantum Electron. 51(9), 310-316 (2019)

Badraoui, N., Berceli, T.: Crosstalk reduction in fiber links using double polarization. Opt. Quantum Electron. 52(4), 1-12 (2020)

Core, M.T.: Cross polarization interference cancellation for fiber optic systems. J. Lightwave Technol. 24(1), 305-312 (2006)

Hayee, M.I., Cardakli, M.C., Sahin, A.B., Willner, A.E.: Doubling of bandwidth utilization using two orthogonal polarizations and power unbalancing in a polarization-division multiplexing scheme. IEEE Photonics Technol. Lett. 13(8), 881-883 (2001)

Johny, J., Shashidharan, S., Sudheer, S.K. Kumar, K.S.: Design and simulation of a radio over fiber system with chromatic dispersion and polarisation mode dispersion compensation. Symposium on Photonics and Optoelectronics, pp. 1-4 (2012)

Morant, M., Pérez, J., Llorente, R.: Polarization division multiplexing of OFDM radio-over-fiber signals in passive optical networks. Opt. Technol. Adv. (2014). https://doi.org/10.1155/2014/269524

Perez, J., Morant, M., Llorente, R., Marti, J.: Joint distribution of polarization-multiplexed UWB and WiMAX radio in PON. J. Lightwave Technol. 27(12), 1912-1919 (2009)

Sekine, K., Sasaki, S., Kikuchi, N.: 10 Gbit/s four-channel wavelength-and polarisation-division multiplexing transmission over $340 \mathrm{~km}$ with $0.5 \mathrm{~nm}$ channel spacing. Electron. Lett. 31(1), 49-50 (1995)

Yao, X.S., Yan, L.S., Zhang, B., Willner, A.E., Jiang, J.: All-optic scheme for automatic polarization division demultiplexing. Optics Epress 15(12), 7407-7414 (2007)

Yoshida, Y., Takami, Y., Inudo, S., Kitayama, K.I., Kanno, A., Yamamoto, N., Kawanishi, T.: On the channel capacity of polarization-multiplexed coherent radio-over-fiber transmissions at millimeter-wave bands. IEEE International Conference on Communications (ICC), pp. 1-6 (2016)

Publisher's Note Springer Nature remains neutral with regard to jurisdictional claims in published maps and institutional affiliations. 